\section{The economic impact of rapid Candida species identification by T2Candida among high-risk patients}

\author{
Sara Pinar Bilir ${ }^{*, 1}$, Cheryl P Ferrufino', Michael A Pfaller ${ }^{2,3}$ \& Julie Munakata
}

\begin{abstract}
Introduction: This study estimates the cost-effectiveness and hospital budget impact of rapid candidemia identification using T2Candida, a novel diagnostic panel with same-day species-specific results. Materials \& Methods: A 1-year decisiontree model estimates hospital costs (2013 US\$) and effects (candidemia-related deaths) for faster diagnostics versus blood culture (BC), accounting for disease prevalence, distribution of Candida species, test characteristics (sensitivity/specificity/time to result), antifungal medication and differential length-of-stay and mortality by appropriate treatment timing. Results: The model estimates a hospital with 5100 annual high-risk patients could possibly save $\$ 5,858,448$ with T2Candida versus BC, a $47.6 \%$ decrease in candidemia diagnosis and treatment budget ( $\$ 1149 /$ patient tested), while averting $60.6 \%$ of candidemia-related mortality. Conclusion: Hospitals may observe lower candidemia-related inpatient costs and mortality with rapid Candida diagnosis.
\end{abstract}

Candida species are a common cause of bloodstream infections, with prevalence ranging from $2-11 \%$ in high-risk patient populations, such as critical care patients and those with compromised immune systems, including oncology and solid organ transplant patients [1,2]. These infections are associated with high mortality and excess inpatient costs. Attributable mortality ranges from 11 to over $47 \%$, depending on time to initiation of antifungal therapy, rising by $>10 \%$ per day of delay in treatment from the time of suspected onset [3,4]. Delay of appropriate treatment is associated with increased hospital length of stay (LOS). Candidemia patients remain in hospital wards significantly longer than patients without candidemia, independent of underlying disease severity or mortality, potentially doubling their LOS [5]. Early identification of candidemia and timely administration of appropriate antifungal therapy is critical to improve outcomes.

Practice patterns for managing high-risk patients vary between institutions. Because of high mortality and costs associated with candidemia, on average $40 \%$ of high-risk patients may be treated with empiric antifungal therapy [6]. However, empiric treatment does not guarantee species-appropriate therapy for affected candidemia patients [3]. Empiric antifungal exposure may impact BC sensitivity in infected patients and also expose a large number of uninfected patients to unnecessary antifungal therapy $[7,8]$. Noncandidemic patients incur unnecessary antifungal treatment costs, while wide-spread use of unnecessary antifungal therapy likely contributes to growing antifungal resistance [8]. For the remaining candidemic patients, appropriate speciesspecific therapy is given only after the blood culture-based diagnostics (BC) isolate a pathogen, which may take at least 3 days due to the slow growth of Candida, increasing mortality and costs [3-5,9].

'Health Economics \& Outcomes Research, 425 Market Street, 7th Floor, IMS Health, San Francisco, CA 94105, USA

${ }^{2}$ T2Biosystems, 101 Hartwell Ave, Lexington, MA 02421, USA

${ }^{3}$ University of lowa College of Medicine \& College of Public Health, lowa City, IA 52242, USA

*Author for correspondence: Tel.: +1 415692 9740; pbilir@us.imshealth.com

\section{KEYWORDS}

- budget impact

- candidemia

- cost-effectiveness

analysis • economic model

- hospital-acquired infection 
To date, no contemporary systematic cost and mortality analysis exists for the management of patients at high risk for candidemia, nor does any study account for the budget impact of early identification of patients with and without candidemia. Collectively, delays in appropriate antifungal therapy have been shown to result in nearly $\$ 80,000$ of additional hospital costs per privately-insured patient (adjusted to 2013 currency) [10]. As payers frequently reimburse hospitals by admission diagnosis, hospitals typically bear these excess candidemia-related costs. Given the high burden of candidemia, a diagnostic strategy for early identification of suspected candidemia patients would be a valuable step toward improving outcomes [11]. The T2Candida panel is the first diagnostic test to provide species-specific Candida detection and identification directly from whole blood (WB) in 3-5 h, without the requirement for prior growth of the organism and with a limit of detection as low as only one colony-forming unit (CFU) per milliliter of WB [12]. The T2Candida panel runs on the T2Dx benchtop instrument utilizing T2 magnetic resonance (T2MR) technology [12]. To evaluate the potential value of this new technology, an economic model was developed to estimate economic consequences of using the T2Candida panel as an adjunct to the current $\mathrm{BC}$ diagnostic strategy in a high-risk hospital patient cohort.

\section{Materials \& methods}

\section{- Model overview}

Cost-effectiveness and budget impact analyses were conducted using a decision-tree model developed in Microsoft Excel (Microsoft Corporation, Redmond, Washington, USA). This model calculates the economic consequences of adopting a new T2Candida diagnostic strategy compared with using $\mathrm{BC}$ alone over 1 year in a hospital setting. The budget impact and cost-effectiveness analyses share a calculation structure, differing only in the types of outcomes estimated. The model assumes that T2Candida is performed as an adjunct test to $\mathrm{BC}$ when Candida is considered a possible diagnosis, and therefore the number of blood cultures remains constant in the BC diagnostic strategy and the T2Candida diagnostic strategy; however, this may not be the case in realworld practice as T2Candida is indicated to be run independent of blood culture. Test-specific sensitivity and specificity are used to estimate the distribution of Candida species and time to species identification, which then drive costs of treatment, hospital LOS and mortality per strategy. A hospital perspective is taken, and costs are reported in 2013 US\$, using the medical CPI [13] as needed to adjust literature-based costs. The budget impact analysis estimated the total annual hospital cost and cost per patient for all tested patients and for patients with confirmed candidemia, as well as net annual hospital cost and net cost per patient. Outcomes in the cost-effectiveness analysis include total costs for the T2Candida panel and BC arms, and candidemia deaths per arm. A 1-year time horizon was chosen to reflect the acute nature of the disease and is consistent with US Modeling Guidelines [14]. One-way sensitivity analyses were conducted on all model variables.

'High-risk', symptomatic patients enter the model, undergoing the $\mathrm{BC}$ strategy (baseline scenario/comparator) or the T2Candida strategy (projected scenario/intervention) (Figure 1). Using sensitivity and specificity data from the literature [12] and a recently completed clinical trial [15], each patient's results are classified as either true positive (TP), false negative (FN), true negative $(\mathrm{TN})$ or false positive $(\mathrm{FP})$ and costs attributed by classification. All patients receive equivalent $\mathrm{BC}$ costs, regardless of outcome; additional diagnostic costs were not included to permit examination of other costs. Candidemia patients (TP and FN) accrue daily hospital costs associated with diagnosis-time dependent LOS, capturing the impact of differential time to appropriate therapy. Daily hospital costs include all facets of care, including pharmacy costs, nursing costs, monitoring tests and labs, and thus no additional medication costs are assigned. Costs for fatal candidemia cases reflect the higher average cost for a fatal hospital stay based on a published comparison [16].

Hospital costs for noncandidemia patients (TN and FP) are assumed to be attributable to underlying disease and are, therefore, not relevant to this study. The cost of antifungal therapy, however, is calculated according to specific dosing for noncandidemic patients to capture any applicable costs of excess empiric antifungal treatment (TN), or unnecessary targeted treatment for FPs. Empiric antifungal treatment is assumed to occur after at least $24 \mathrm{~h}$ 


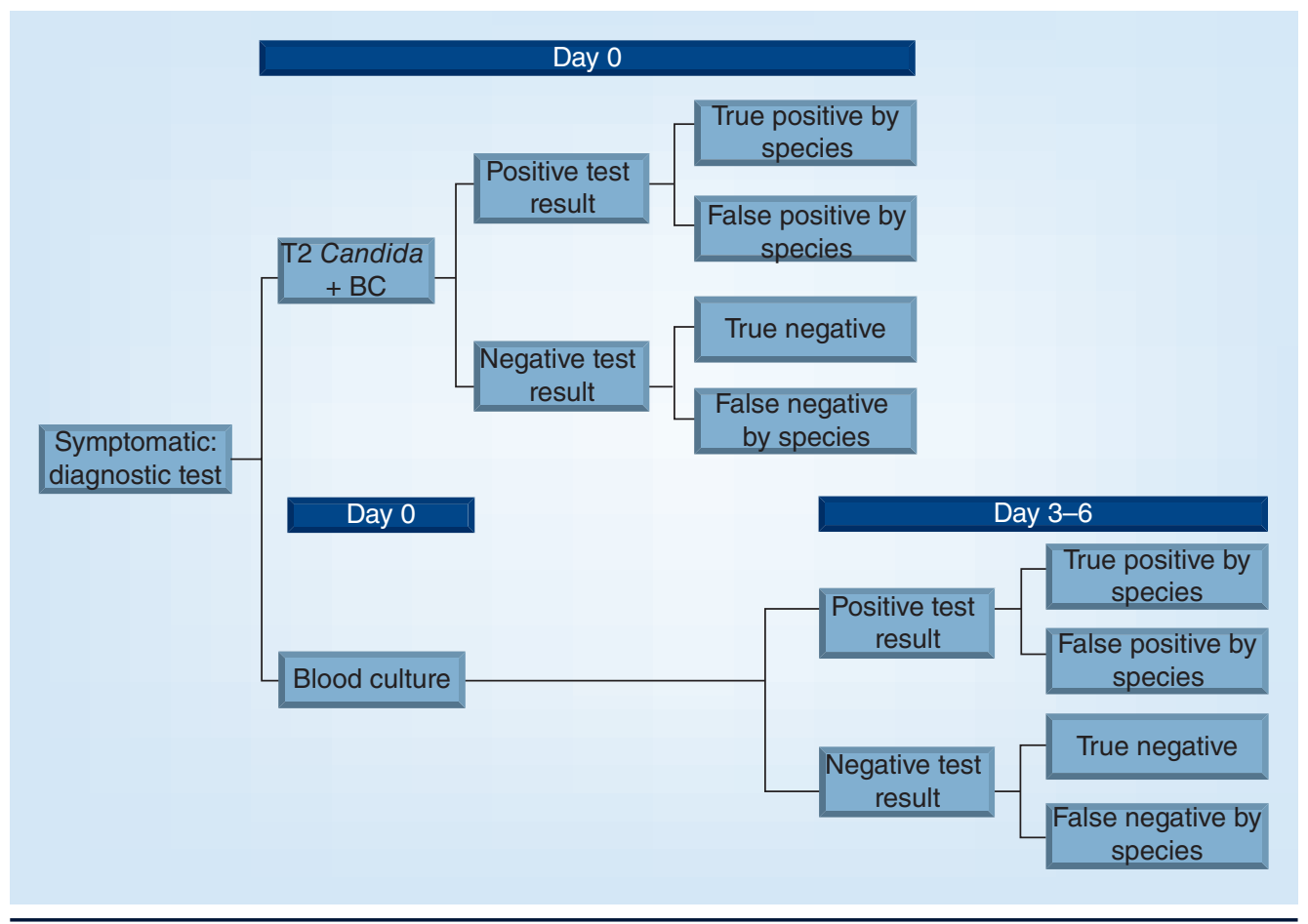

Figure 1. Model structure.

BC: Blood culture.

postinitial BC, reflecting common practice to start with broad-spectrum antibiotics followed by antifungal coverage. This assumption is tested in sensitivity analysis.

\section{- Inputs \& assumptions}

Data sources

Data inputs in the model were derived from many sources including peer-reviewed literature, and an analysis of hospital charge data performed using the IMS Hospital Charge Data Master (CDM) Database. This database contains detailed hospital stay data collected annually for approximately 9 million inpatient encounters from over 650 hospitals. A retrospective cohort analysis of inpatient hospital billing records from 01 January 2011 through 30 September 2013 was conducted for patients $18+$ years old with a primary or secondary candidemia admission or discharge diagnosis (ICD-9 112.5,112.89, 112.9), at least one diagnostic test such as a BC, and evidence of antibiotic or antifungal therapy to estimate candidemia-associated costs. Patients were excluded if admitted from or discharged to another hospital or acute care facility. A total of 3891 cases met inclusion criteria, of which $88.3 \%$ were diagnosed by BC. The CDM data, reported as charges, were converted using a cost-to-charge ratio derived from the publicly available Healthcare Utilization Project (HCUP) dataset [17].

\section{Population \& clinical variables}

The annual number of 'high-risk' admissions within a single 500 bed hospital is assumed to be approximately 5100 patients in the base case [18]. Common 'high-risk' patient populations include those with solid tumors, hematological malignancy, critical care admissions and transplant patients (solid organ, bone marrow, hematopoietic stem cell). Disease prevalence and Candida species distributions are then applied. A 3\% prevalence was used as default, at the low end of values found throughout the country (1). The species distribution was estimated for five clinically-relevant Candida species, comprising $>97 \%$ of all Candida infections, using weighted estimates from large surveillance registries (Table 1) [19,20].

Time to antifungal treatment initiation is a key driver of patient outcomes such as LOS and mortality [3-5,9]. In the default scenario, the total LOS for patients in whom appropriate antifungal therapy is initiated within $24 \mathrm{~h}$ is 7 days [5]. Approximately half of patients will 


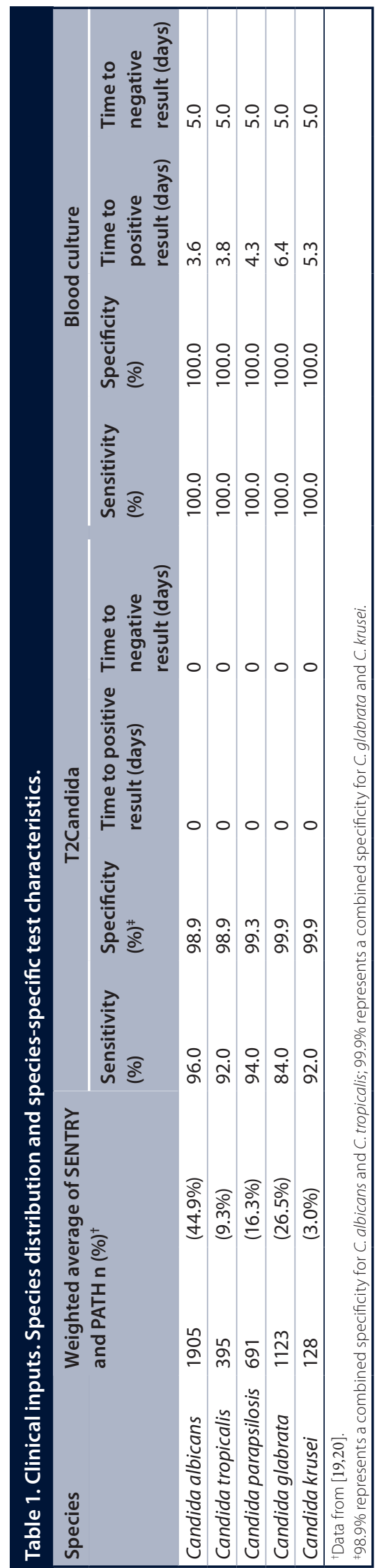

be diagnosed in the ICU, in which 4.4 days will be spent, and the remaining 2.6 days will be in a non-ICU setting [5]. Patients who experience $1+$ days of delay in initiation of antifungal therapy have an estimated LOS of 15.8 days, 7.7 in the ICU and 8.1 in a non-ICU setting [5]. The remaining $50 \%$ accrue the same number of inpatient days, but in a non-ICU setting only. Antifungal therapy delays result in significantly higher mortality, with rates more than doubling after a delay of one day [3,4]. Mortality for patients in whom antifungal therapy is initiated within $12 \mathrm{~h}$ is $11.1 \%$, increasing to 24,37 and $41 \%$ for patients with treatment initiated on days 1,2 , or $\geq 3$, respectively $[3,4]$. According to an analysis using HCUP data, hospital costs for patients who die are approximately 2.7 times higher than for survivors [16]. This value is used to multiply hospital costs for candidemia patients who die in either strategy, assuming that fatal candidemia cases should have equivalent costs regardless of the timing of diagnosis.

\section{Diagnostic test characteristics}

Sensitivity and specificity estimates were applied across the tested population (Table 1). BC was conservatively assumed to have $100 \%$ specificity and sensitivity, despite the reported value of $50 \%$ sensitivity for the diagnosis of invasive candidiasis [25]. Test-specific time to negative result is used as a proxy for the duration of empiric therapy administered to a noncandidemic patient. The time to positive result is based on time to species identification among different diagnostic approaches, and dictates when appropriate therapy begins for positive patients. T2Candida results are returned in 3-5 h (either positive or negative), including the species-level identification of Candida spp. [26]. To achieve the same details from $\mathrm{BC}$ requires 3.6-6.4 days, for detection of the organism and species identification by conventional methods [9]. It is assumed that a negative $\mathrm{BC}$ test result is returned in 5 days. Similarly, the model assumes that FP patients receive antifungal therapy for 5 days corresponding to the time to a negative $\mathrm{BC}$ result.

\section{Treatment assumptions}

Although time to species identification is a proxy for treatment initiation, the model also assumes any empiric antifungal therapy begins on day 1 . These patients experience the shorter LOS and mortality associated with earlier treatment if 
medication is appropriate by species. In clinical practice, fluconazole or an echinocandin may be used prior to receipt of BC results, based on published guidelines [24]. However, current practice patterns suggest that echinocandins are in greater use due to increasing resistance to azoles [8-9,20,27]. All five Candida species are considered to be susceptible to micafungin, and thus it is the default targeted therapy for all species, and administered empirically to $40 \%$ of patients in the BC arm prior to results in the default analysis. Patients on the T2Candida strategy do not need empiric therapy due to early results, and receive appropriate therapy within the first $12 \mathrm{~h}$ of testing even though the time to result suggests that appropriate therapy could be administered in 3-5 h (data to show cost and mortality savings for intervention earlier than $12 \mathrm{~h}$ are not available) [3-5]. In patients receiving fluconazole, therapy was considered to be appropriate for treatment of $C$. albicans, $C$. tropicalis and $C$. parapsilosis and inappropriate for C. glabrata and C. krusei [9,26]. Appropriate targeted therapy is initiated on the day of species identification in patients diagnosed by BC but not given empiric therapy. With these assumptions, pharmacy costs associated with unnecessary empiric antifungal therapy are estimated for noncandidemia patients. Lack of susceptibility to antifungal treatments should be equivalent across the population (nondifferential costs by diagnostic method), and is omitted from the model.

\section{Resource utilization \& costs}

Use of $\mathrm{BC}$ is assumed to be equivalent between strategies (4 BCs taken as two serial draws over 2 days) in the base case, and while the use of the T2Candida assay is assumed, the cost is omitted to permit this analysis to estimate the cost differentials from other input sources. Unit costs include BC costs, daily hospital costs and empirical drug costs (Table 2).

To estimate the inpatient stay cost, per diem costs for ICU and non-ICU are based on published values [19-21,28], and applied to the LOS. Default treatment dosing assumptions for each species of Candida follow published guidelines [9,26]. Drug costs are calculated using recommended dose quantity, formulation and vial size, using wholesale acquisition costs (WAC) from the Medispan PriceRx online database [23].

A summary of model assumptions is listed in Box 1.

\section{Results}

\section{- Base case results}

In a hospital admitting 5100 'high-risk' patients per year, about 153 patients will be diagnosed with candidemia, assuming a $3 \%$ prevalence rate. Switching from the current gold standard BC diagnostic strategy to a T2Candida-based strategy is estimated to result in potential annual savings of $\$ 5,858,448$ (47.6\%). The annual cost for testing and treating the high-risk patients with the baseline BC strategy is estimated at $\$ 12,298,598$ compared with $\$ 6,440,150$ in the projected T2Candida strategy, decreasing the cost per tested patient from $\$ 2411$ to $\$ 1263$ when adopting an institution-wide T2Candida testing strategy for a potential savings of $\$ 1148$ per tested patient. Savings were predominantly due to the shortened hospital stay for survivors and lower

\section{Table 2. Cost inputs.}

\begin{tabular}{|c|c|c|c|}
\hline Variable & Default value (US\$) & Source & Ref. \\
\hline Cost per day: non-ICU & $1,560.00$ & Golan 2005 converted to 2013 US\$ & [21] \\
\hline Cost per day: ICU & $4,140.84$ & Dasta 2005 converted to 2013 US\$ & [22] \\
\hline Therapy & Mean daily cost per regular dose (US\$) & Source & Ref. \\
\hline Fluconazole & 16.34 & \multirow{7}{*}{$\begin{array}{l}\text { All daily costs are calculated based on recommended } \\
\text { dose }{ }^{24} 9 \text { and WAC price. Additional costs associated } \\
\text { with loading doses were added as necessary. }\end{array}$} & \multirow[t]{7}{*}[23,24]{} \\
\hline Itraconazole & 14.84 & & \\
\hline Anidulafungin & 180.00 & & \\
\hline Caspofungin & 350.88 & & \\
\hline Micafungin & 187.00 & & \\
\hline Amphotericin B & 76.00 & & \\
\hline Abelcet (lipid formulation) & $3,885.00$ & & \\
\hline
\end{tabular}




\section{Box 1. Model assumptions.}

\section{Time to initial therapy}

- All patients start antifungal therapy at the time of a positive diagnostic test.

- Due to the rapid results of T2Candida, it is assumed that all patients will be initiated on antifungal therapy within hours of the initial diagnostic test (Day 0).

- For blood culture (BC)-tested patients, species-specific timing dictates the number of days between the test and the initiation of therapy. Because no data exist to capture the benefit of treating within less than $12-24 \mathrm{~h}$ of initial test, daily increments in time to initiation are employed.

- For patients who do not receive an immediate diagnosis, approximately $40 \%$ will start on empiric antifungal therapy on Day 1 (prior to test results).

\section{Diagnostic tests}

- Each initial T2Candida test is performed in conjunction with the standard BC testing regimen (four BCs taken as two serial draws over 2 days).

- The cost of BC tests include speciation costs (e.g., labor) and supply costs (e.g., vials), and include two vials each (one aerobic, one anaerobic).

\section{Treatment assumptions}

- Empiric therapy: In this model, empiric therapy is defined as therapy administered prior to either a positive or negative test result and/ or Candida speciation. Patients who do not receive an immediate test result and initiate empiric antifungal therapy will begin medication on Day 1. Total medication costs associated with therapy will only be calculated for patients with no diagnosed candidemia. Although empiric therapy could be appropriate for patients initiating antifungal therapy to which their Candida species is susceptible, this model assigns daily hospital costs to patients with true candidemia, including all pharmacy costs, and thus these additional costs are not captured separately in the model.

- After the test result is returned, the false positive patients will receive 'targeted' treatment. Targeted therapy for true positive patients will be included in daily hospital costs, and thus not separately calculated.

- It is assumed that appropriate treatment for Candida albicans, Candida tropicalis, Candida parapsilosis, Candida glabrata and Candida krusei is an echinocandin (micafungin as default).

- The default settings assume that patients will begin micafungin as empiric or targeted therapy for all species.

- No treatment-related side effects are included in the model, as recent first-line medications have less severe side effect profiles than were previously available.

\section{Resource use \& costs}

- All costs are reported in 2013 US\$.

- Costs are estimated according to candidemia diagnosis status. For candidemia patients, costs are estimated according to daily hospital costs to capture the benefit of a shorter LOS due to early diagnosis and treatment. It is assumed that 50\% of patients receive some ICU care followed by care in another ward, while the remaining patients are treated exclusively out of the ICU setting. All candidemia patients experience the same average number of days in the hospital, regardless of ward.

- Noncandidemia patients accrue itemized costs associated with unnecessary treatment. For medications administered via single-dose vials, costs may include some wastage. Multiuse vial drug costs are calculated at the unit level (e.g., $\$ / \mathrm{mg}$ ) and assume no wastage.

- Patients who die of candidemia accrue a 2.7-times higher cost per hospital stay compared with BC patients who survive.

hospital costs associated with the lower mortality rates in the T2Candida strategy (Table 3).

The estimated potential savings per patient with candidemia is $\$ 26,887$, a $48.8 \%$ reduction in hospital costs (Table 3). Rapid species diagnosis and earlier treatment associated with the T2Candida strategy results in fewer deaths, reducing overall mortality by 31.7 deaths $(60.6 \%$; BC: 52.3 deaths; T2Candida: 20.6 deaths). Given these improvements, a diagnostic strategy including T2Candida would be considered dominant over BC alone in cost-effectiveness analysis.

\section{- Sensitivity analysis}

Univariate sensitivity analysis showed that aside from test sensitivities and specificities, the model was most sensitive to the time to positive results for the two most common Candida species (C. albicans and $C$. glabrata) using each test strategy, and costs accrued in the non-ICU setting (Figure 2 shows the impact on results of varying parameter values upwards or downwards for the ten most sensitive parameters). However, the direction of results remained robust under all tested scenarios.

Empiric treatment with fluconazole may decrease the net savings to $\$ 4,169,938$, a $39.3 \%$ reduction in savings relative to baseline. The decrease in savings reflects the added costs of inappropriately treated uninfected patients despite the cheaper daily cost of fluconazole.

The sensitivity of LOS was also tested. If early treatment shortens ICU time but not 


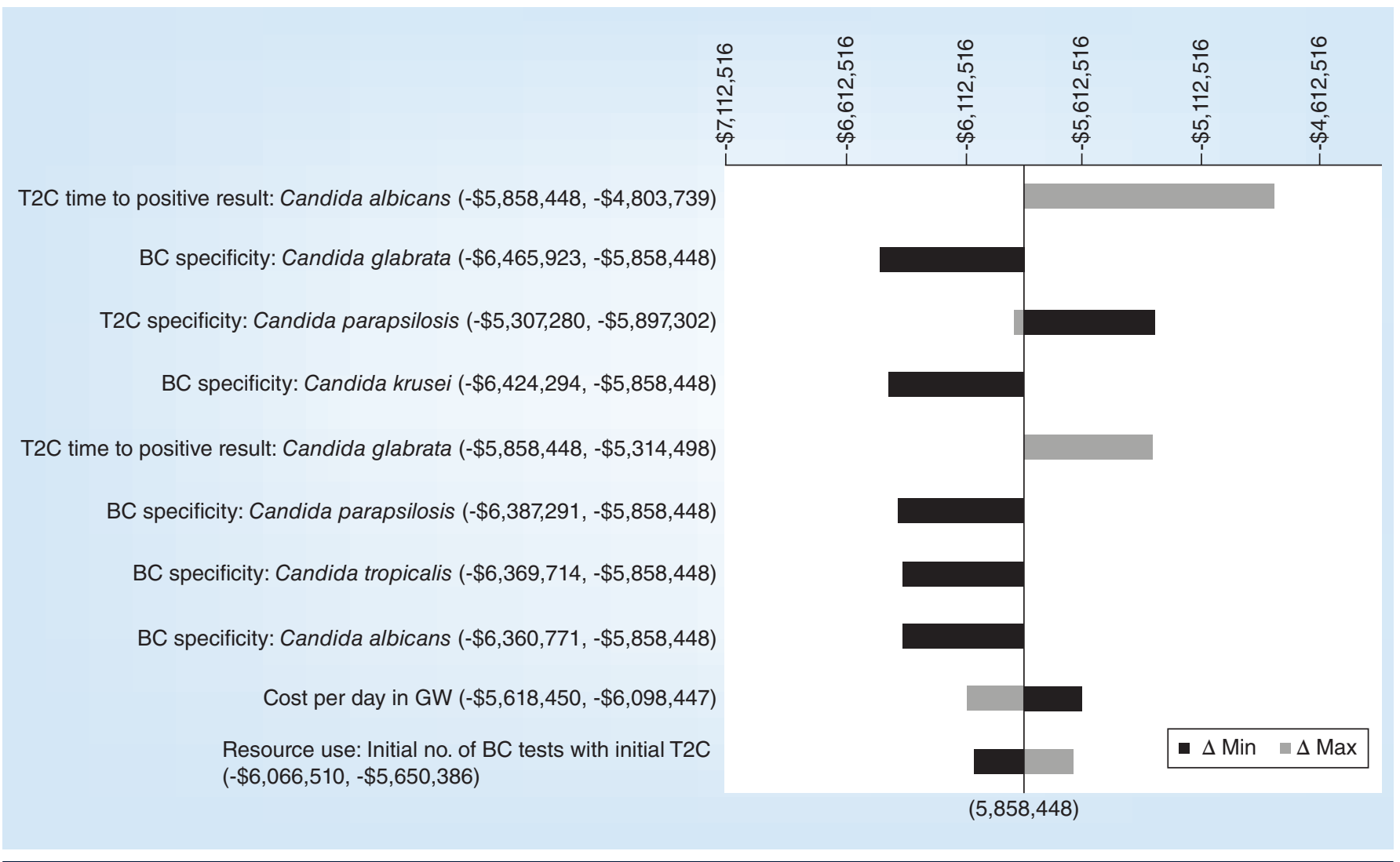

Figure 2. One-way sensitivity analysis tornado diagram: ten most sensitive parameters. Note that BC sensitivity and specificity is assumed perfect in the base case, and therefore can only be analyzed at lower values in univariate analysis. BC: Blood culture; GW: General ward at the hospital; T2C: T2Candida.

the overall LOS (15.8 days overall: 4.4 days ICU, 11.4 days non-ICU), the potential savings are $\$ 812$ per tested patient (33.7\%), or $\$ 15,659$ per candidemic patient. Exploration of the required LOS values to lose cost-savings altogether (breakeven analysis), showed that because early diagnosis prevents empiric treatment costs, the LOS must actually be longer for patients diagnosed early in order to lose cost-savings; there is no data to suggest an optimal distribution of days in ICU and nonICU in an exploratory setting, but with the original assumption that $50 \%$ of the population spends no time in the ICU, and with an assumption that among the $50 \%$ who do, $50 \%$ of their time is spent there regardless of diagnosis time, the LOS must be approximately twice as long for early diagnosed patients to lose the cost savings.

Because costs for patients who die are an important potential source of savings with T2Candida due to the lower mortality rate associated with early diagnosis and therapy, sensitivity analysis tested lower costs per fatal hospital stay. If a fatal stay costs only two times more than a survivor's stay, rapid detection of candidemia with T2Candida maintains considerable potential savings of $\$ 998$ (46.2\%) per tested patient or $\$ 21,874$ (46.7\%) per candidemic patient.

Although base case analysisis focused on understanding the economic impact of the rapid diagnosis of Candida, if we account for the potential cost of the T2Candida test and capital costs for the testing instrument, the model consistently demonstrates cost-savings associated with rapid Candida diagnosis. Assuming a list price of $\$ 265$ per T2Candida test, the total savings are offset by $\$ 1,351,500$ in test costs and would result in a net savings of $\$ 4,506,948$ per year or $\$ 884$ per tested patient. When additionally considering capital costs of $\$ 150,000$ per instrument, assuming two instruments per facility, an estimated savings of $\$ 825$ per tested patient is realized in the first year. Assuming an equal distribution of 
Table 3. Base case results.

\begin{tabular}{lllll} 
Cost per tested patient & T2Candida strategy (US\$) & Blood culture strategy (US\$) & Absolute difference (US\$) & Relative difference (\%) \\
\hline Diagnostic test cost & 407.96 & 407.96 & 0.00 & 0.0 \\
\hline Antifungal treatment & 20.68 & 362.78 & -342.10 & -94.3 \\
Hospital stay & 834.13 & $1,640.74$ & -806.61 & -49.2 \\
\hline $\begin{array}{l}\text { Total costs } \\
\text { Cost per Candidemia patient }\end{array}$ & $1,262.77$ & $2,411.49$ & $-1,148.72$ & -47.6 \\
\hline $\begin{array}{l}\text { Diagnostic test cost } \\
\text { Antifungal treatment }\end{array}$ & 407.96 & & & 0.0 \\
Hospital stay & 0.00 & 407.96 & 0.00 & 0.0 \\
\hline Total costs & $27,804.37$ & 0.00 & 0.00 & -49.2 \\
\hline
\end{tabular}

patients over the course of the year, savings could exceed capital costs associated with running the T2Candida test in the first month.

Potential savings due to T2Candida also remain quite high when testing the assumption around empiric therapy. Clinicians may be reluctant to stop a course of treatment once started, in which case empirically treated patients may receive up to 14 days of antifungal medication. This scenario would lead to potential savings of $\$ 9,188,759$ (58.8\%) per year or $\$ 1802$ per tested patient with a full course of treatment. If empiric therapy is initiated on day 0 rather than day 1 but given only according to the original 5-day assumption before negative results are revealed, the potential savings from using the T2Candida strategy would be $\$ 4,521,081(42.8 \%)$ in total costs or $\$ 886$ per tested patient. Similar results are found with combinations of drug choice, timing and proportion of 'high-risk' patients treated empirically (Figure 3). As the proportion of patients receiving empiric therapy increases, savings increase with micafungin treatment due to high drug cost. Savings fall with fluconazole treatment administered empirically to increasing proportions of patients due to the cheaper drug cost, although for inappropriately treated patients, mortality will also rise.

\section{Discussion}

Because of the significant mortality associated with Candida infections [29], many hospital systems have implemented empiric treatment strategies to manage patients at risk for these infections; although candidemia incidence is low, over $40 \%$ of symptomatic high-risk patients may receive empiric therapy [6]. Despite this aggressive patient management strategy, inappropriate dosing is common, and promotes resistance $[8,21]$. These more aggressive practices also fail to improve the mortality rate for Candida-related sepsis [4].

This study demonstrates that T2Candida, a diagnostic panel to detect and identify Candida species in 3-5 h directly from whole blood, has the potential to significantly reduce costs and mortality rates in patients at high risk for candidemia. Using an economic model to quantify the potential economic and clinical outcomes associated with utilizing the T2Candida panel as a primary diagnostic tool in a hospital's high-risk population shows that diagnosing patients sooner, prompting earlier targeted therapy with resultant decreases in mortality and LOS, may dramatically decrease hospital costs. The application of species-specific therapy enabled by rapid Candida identification demonstrated a potential savings of over 30 lives per year in a typical hospital setting, corresponding to a $60.6 \%$ reduction in mortality. Coupling this mortality reduction with cost savings shows that a T2Candida diagnostic strategy may markedly improve the management of candidemia compared with the standard of care BC strategy.

Faster diagnosis and species identification could mitigate the need for empiric antifungal therapy and permit immediate targeted antifungal therapy, while the current BC strategy requires either waiting for conclusive results or initiating empiric therapy on all high-risk patients. Also, although $\mathrm{BC}$ sensitivity and specificity were set to $100 \%$ in the model, real world data indicates that sensitivity may be as low as $50 \%$ [25], leading to a prolonged duration before appropriate treatment. The model demonstrates that the cost of administering empiric therapy to a broader uninfected patient population may be substantial, irrespective of the costs associated with the growing issue of resistance, which could be significant. Of the 


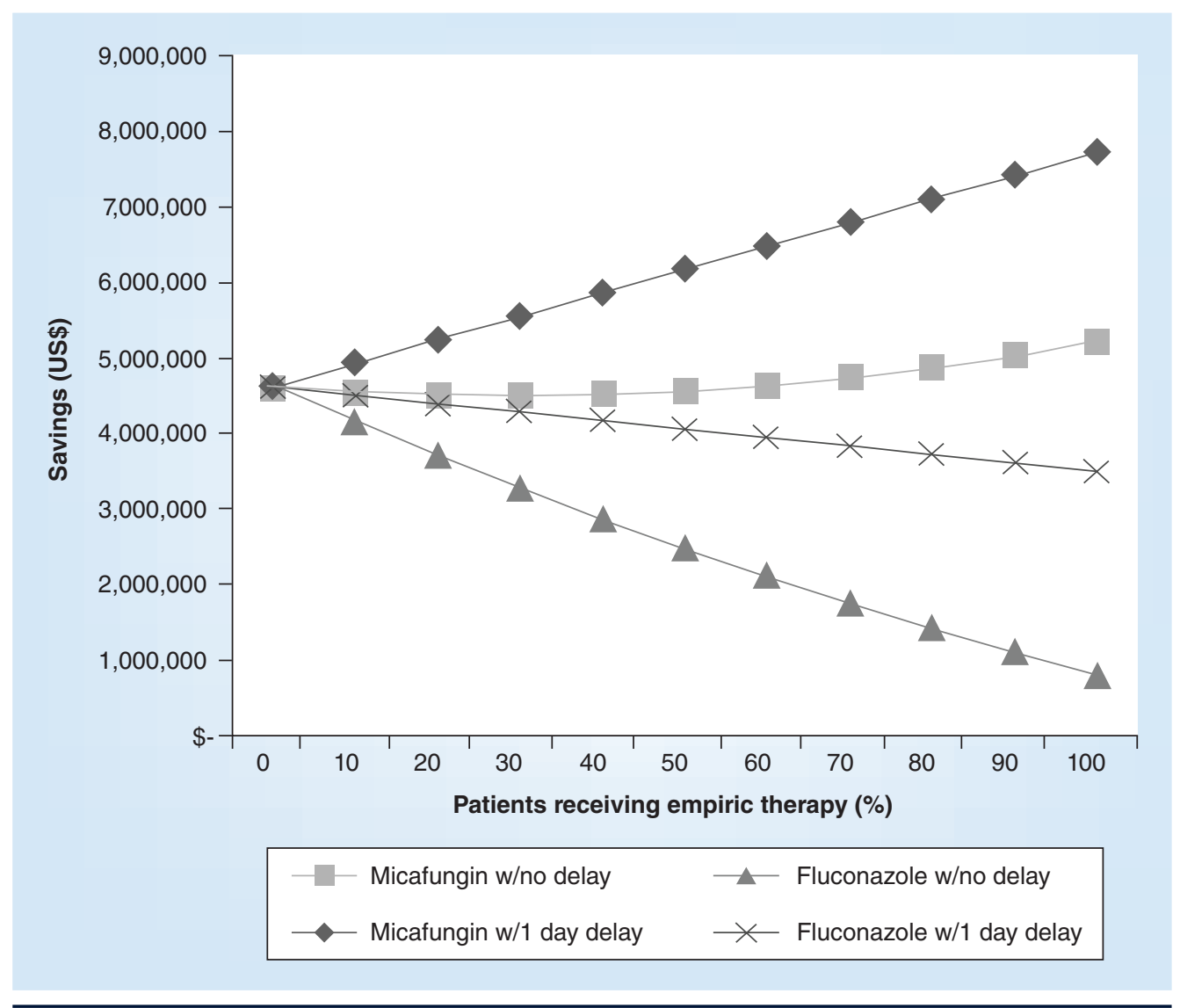

Figure 3. Impact of empiric therapy choice and timing. w/: With.

$\$ 5.86 \mathrm{M}$ total potential savings, approximately $\$ 1.74 \mathrm{M}(29.7 \%)$ accrue to noncandidemic patients.

Several studies have looked at the cost burden of candidemia, often comparing candidemia patients to noncandidemia patient controls. However, the present study contributes to the literature in two areas: it estimates the costs associated with the BC standard compared with a new, faster diagnostic strategy; and it broadens the economic analysis to include the direct hospital costs of managing patients at risk for candidemia, as opposed to only those diagnosed as candidemic. The clinical and financial impact of delayed treatment has been established [5,21]. In 2005, Zaoutis estimated the excess cost burden for a candidemia patient to be as high as \$39,331 compared with noncandidemic patients [30], while Rentz found costs exceeded $\$ 44,000$ in 1998 ; in 2013 currency this is nearly $\$ 80,000$ [10]. This model estimates that current LOS-related costs associated with diagnosis by BC may exceed
$\$ 55,000$ per stay per candidemic patient, nearly $\$ 27,000$ more than the costs for a candidemic patient treated following the earliest possible diagnosis. It is important to understand that improvements in antimicrobial management, LOS, mortality and healthcare costs attributed to more rapid diagnostic testing are contingent on rapid result communication directly to the treating physician or designee such as an infectious disease-trained pharmacist or member of the antimicrobial stewardship team [31,32]. Without real-time notification and/or administrative support and intervention, none of the potential benefits of rapid diagnostic testing will be realized [33].

This study focused on the potential impact of LOS, reduction in the excess antifungal treatment and improved survival. However, a number of additional aspects of care, omitted from the model due to lack of sufficient data, may also have additional fiscal consequences within hospitals. Lower use of empiric therapy will avert medication side effects, and could 
slow antimicrobial resistance in the long run. Ruling out Candida earlier may also accelerate administration of more appropriate empiric therapy for underlying bacterial infections. In candidemic patients, early treatment may truly resolve more infections prior to discharge, reducing readmissions, while quicker infection resolution may reduce or avert reimbursement penalties for nosocomial infections. Finally, the impact on improved quality of life for the patient population was not quantified in this model.

Our study has several limitations. This model uses a variety of assumptions and data taken from the contemporary literature and thus may not be representative of all clinical and diagnostic settings. It should be noted, however, that the model was developed with a fully flexible framework for the purpose of testing these assumptions, and such tests have been performed in sensitivity analysis. We recognize that per diem hospital costs likely vary between hospitals [21,22]. We selected values from the literature as a representative average, but true savings may differ by site. Further, practice patterns vary, impacting the proportion of a population receiving empiric therapy, what empiric medication is used, and when it is initiated. Sensitivity analyses have examined the potential change in costs associated with using the cheapest antifungals, and initiating empiric medication earlier and in different proportions of the population. However, overall findings that early diagnosis results in cost savings remain true under even significantly more conservative assumptions than comprise the base-case analysis on these points. The actual sensitivity of the T2Candida Panel has not been fully characterized in all clinical situations such as those surrounding intraabdominal candidiasis [25]; however, studies with blinded samples from patients with candidemia [12] and the results from the pivotal 1801 patient clinical trial [15] clearly support its role as an adjunct to BC in the diagnosis and management of candidemia. We have used data from two multicenter surveillance surveys to estimate the potential species distribution of Candida causing more than $97 \%$ of candidemia in the USA $[19,20]$ but realize that this distribution may vary from institution to institution depending on antifungal use practices and other factors that may affect the local epidemiology. Having said this, additional population-based and sentinel surveillance studies of Candida species by geographic region [34-36] provide ample support for the rank order of the Candida species shown in Table 1 as representative of that seen in US hospitals. Finally, although the model does not take into account prior or concurrent antibiotic or antifungal therapy, Morrell et al. have shown that patients with prior antibiotic therapy have an increased mortality risk [4]. Further testing and evidence from real-world use of the T2Candida assay will provide opportunities for future research to validate or further inform the overall costs and cost-effectiveness of early diagnosis in this population.

\section{Conclusion}

As the regulatory environment changes, hospitals continue to look for ways to minimize liability from bloodstream infections that cause sepsis. For high risk patients, these strategies rely on the aggressive administration of antifungal therapy in the absence of microbiological evidence for Candida, which is costly, increases resistance and fails to effectively treat all species. Identification of patients with and without candidemia in mere hours has the potential to significantly decrease resource utilization throughout the hospital and overall healthcare system, while reducing the mortality rate from 40 to as low as 11\% [4]. While significant literature exists to support the reduction of costs and mortality as a result of the early identification of candidemia patients, no study has captured the impact at time points under $12 \mathrm{~h}$. Although savings/cost-avoidance at a hospital level may vary depending on hospital contracting for echinocandins, volume of candidemia and effectiveness of stewardship interventions, and even the addition of costs to cover the T2Candida panel, savings are still likely to be substantial [18]. Given the data from existing studies, it is reasonable to conclude that more rapid delivery of appropriate care would further improve mortality and economic savings beyond those modeled here. Innovative diagnostic technologies such as T2Candida have the potential to generate improved clinical outcomes and significant hospital savings.

\section{Financial \& competing interests disclosure}

The study was supported by T2 Biosystems. MA Pfaller is an employee of T2 Biosystems. The authors have no other relevant affiliations or financial involvement with any organization or entity with a financial interest in or financial conflict with the subject matter or materials discussed in the manuscript apart from those disclosed.

No writing assistance was utilized in the production of this manuscript. 


\section{EXECUTIVE SUMMARY}

- Growth-based detection of Candida among high risk patients can delay treatment decisions by as much as 3-6 days, with longer hospitalization and higher mortality.

- As the regulatory environment changes, hospitals continue to look for ways to minimize liability from bloodstream infections that cause sepsis.

- For high risk patients, these strategies rely on the aggressive administration of antifungal therapy in the absence of microbiological evidence for Candida, which is costly, increases resistance, and fails to effectively treat all species.

- Faster diagnosis with species-specific results in 3-5 h could reduce patient mortality and management costs.

- Using an MS Excel 1-year decision-tree model to estimate hospital costs and effects (candidemia-related deaths) of using faster diagnostics versus BC alone, a typical hospital with 5100 annual high-risk patients could save as much as $\$ 5,858,448$ in total hospital costs.

- This is a $47.6 \%$ decrease in a candidemia diagnosis and treatment budget ( $\$ 1149$ per patient tested), prior to accounting for the cost of the faster diagnostic test.

- Across all patients tested, faster diagnosis would avert 31.7 deaths, or $60.6 \%$ of candidemia-related mortality per hospital.

- Cost savings remain robust with alternate inputs or assumptions.

- A significantly faster diagnostic method than BC-based diagnostics can be beneficial to a hospital, potentially decreasing candidemia-related inpatient hospital costs and mortality.

- Additional costs that would be averted, but remain uncalculated, include reduced antimicrobial resistance, avoiding resetting therapeutic courses, along with averted diagnostic and therapeutic interventions.

\section{References}

Papers of special note have been highlighted as:

- of interest; $\bullet$ of considerable interest

1 Ostrosky-Zeichner L, Sable C, Sobel J et al. Multicenter retrospective development and validation of a clinical prediction rule for nosocomial invasive candidiasis in the intensive care setting. Eur. J. Clin. Microbiol. Infect. Dis. 26, 271-276 (2007).

2 Eggimann P, Bille J, Marchetti O. Diagnosis of invasive candidiasis in the ICU. Ann. Intensive Care 1, 37 (2011).

3 Garey KW, Rege M, Pai MP et al. Time to initiation of fluconazole therapy impacts mortality in patients with candidemia: a multi-institutional study. Clin. Infect. Dis. 43, 25-31 (2006)

- This retrospective cohort study demonstrates that a delay in the initiation of fluconazole therapy in hospitalized patients with candidemia significantly impacted mortality.

4 Morrell M, Fraser VJ, Kollef MH. Delaying the empiric treatment of candida bloodstream infection until positive blood culture results are obtained: a potential risk factor for hospital mortality. Antimicrob. Agents Chemother. 49, 3640-3645 (2005).
5 Arnold HM, Micek ST, Shorr AF et al. Hospital resource utilization and costs of inappropriate treatment of candidemia. Pharmacotherapy 30, 361-368 (2010).

-• This retrospective cohort study demonstrates differential length of hospital stay based on whether treatment for candidemia is received within $24 \mathrm{~h}$.

6 Valerio M, Rodriguez-Gonzalez CG, Munoz $\mathrm{P}$ et al. Evaluation of antifungal use in a tertiary care institution: antifungal stewardship urgently needed. J. Antimicrob. Chemother. 69, 1993-1999 (2014).

7 Riedel S, Eisinger SW, Dam L et al. Comparison of BD Bactec Plus Aerobic/F medium to VersaTREK Redox 1 blood culture medium for detection of Candida spp. in seeded blood culture specimens containing therapeutic levels of antifungal agents. J. Clin. Microbiol. 49, 1524-1529 (2011).

8 Pfaller MA. Antifungal drug resistance: mechanisms, epidemiology, and consequences for treatment. Am. J. Med. 125, S3-S13 (2012).

9 Fernandez J, Erstad BL, Petty W et al. Time to positive culture and identification for Candida blood stream infections. Diagn. Microbiol. Infect. Dis. 64, 402-407 (2009).
10 Rentz AM, Halpern MT, Bowden R. The impact of candidemia on length of hospital stay, outcome, and overall cost of illness. Clin. Infect. Dis. 27, 781-788 (1998).

- Rentz et al. use Healthcare Utilitization Project (HCUP) data to demonstrate the high cost burden associated with candidemia, also specifying that much of that is due to the duration of hospital stay.

11 Alexander BD, Pfaller MA. Contemporary tools for the diagnosis and management of invasive mycoses. Clin. Infect. Dis. 43 , S15-S27 (2006).

12 Neely LA, Audeh M, Phung NA et al. T2 magnetic resonance enables nanoparticlemediated rapid detection of candidemia. Whole Blood Sci. Transl. Med. 5, 182ra54 (2013).

-• This study describes the T2 magnetic resonance approach, and demonstrates the speed with which it can turn around results.

13 Medical Consumer Price Index. www.economagic.com

14 Mauskopf JA, Sullivan SD, Annemans L et al. Principles of good practice for budget impact analysis: report of the ISPOR Task Force on good research practices-budget 
impact analysis. Value Health 10, 336-347 (2007)

15 Mylonakis E, Clancy CJ, Ostrosky-Zeichner $\mathrm{L}$ et al. T2 magnetic resonance assay for the rapid diagnosis of candidemia in whole blood: a clinical trial. Clin. Infect. Dis. 60, 892-899 (2015).

- This paper presents the clinical trial in which the novel rapid diagnostic approach is used as adjunct to blood culture in the diagnosis of candidemia; the current analysis relies on sensitivity and specificity information from this clinical trial.

16 Zhao Y, Encinosa W. The Costs of End-of-Life Hospitalizations, 2007: Statistical Brief \#81.

www.hcup-us.ahrq.gov

17 Healthcare Utilization Project. National Inpatient Sample. www.hcup-us.ahrq.gov

18 Aitken SL, Beyda ND, Shah DN et al. Clinical practice patterns in hospitalized patients at risk for invasive candidiasis: role of antifungal stewardship programs in an era of rapid diagnostics. Ann. Pharmacother. 48, 683-690 (2014).

19 Pfaller MA, Moet GJ, Messer SA et al. Candida bloodstream infections: comparison of species distributions and antifungal resistance patterns in community-onset and nosocomial isolates in the SENTRY Antimicrobial Surveillance Program, 2008-2009. Antimicrob. Agents Chemother. 55, 561-566 (2011).

20 Pfaller MA, Neofytos D, Diekema D et al. Epidemiology and outcomes of candidemia in 3648 patients: data from the Prospective Antifungal Therapy (PATH Alliance(R)) Registry, 2004-2008. Diagn. Microbiol. Infect. Dis. 74, 323-331 (2012).

21 Golan Y, Wolf MP, Pauker SG et al. Empirical anti-Candida therapy among selected patients in the intensive care unit: a cost-effectiveness analysis. Ann. Intern. Med. 143, 857-869 (2005).

22 Dasta JF, McLaughlin TP, Mody SH, Piech CT. Daily cost of an intensive care unit day: the contribution of mechanical ventilation. Crit. Care Med. 33, 1266-1271 (2005).

23 Medispan PriceRx. Wolters Kluwer. http://pricerx.medispan.com

24 Pappas PG, Kauffman CA, Andes D et al. Clinical practice guidelines for the management of candidiasis: 2009 update by the Infectious Diseases Society of America. Clin. Infect. Dis. 48, 503-535 (2009).

25 Clancy CJ, Nguyen MH. Finding the "missing 50\%" of invasive candidiasis: how nonculture diagnostics will improve understanding of disease spectrum and transform patient care. Clin. Infect. Dis. 56, 1284-1292 (2013).

26 Beyda ND, Alam MJ, Garey KW. Comparison of the T2Dx instrument with T2Candida assay and automated blood culture in the detection of Candida species using seeded blood samples. Diagn. Microbiol. Infect. Dis. 77, 324-326 (2013).

27 Alexander BD, Johnson MD, Pfeiffer CD et al. Increasing echinocandin resistance in Candida glabrata: clinical failure correlates with presence of FKS mutations and elevated minimum inhibitory concentrations. Clin. Infect. Dis. 56, 1724-1732 (2013).

28 Garey KW, Pai MP, Suda KJ et al. Inadequacy of fluconazole dosing in patients with candidemia based on Infectious Diseases Society of America (IDSA) guidelines. Pharmacoepidemiol. Drug Saf. 16, 919-927 (2007).

29 Kollef M, Micek S, Hampton N et al. Septic shock attributed to Candida infection: importance of empiric therapy and source control. Clin. Infect. Dis. 54, 1739-1746 (2012).

30 Zaoutis TE, Argon J, Chu J et al. The epidemiology and attributable outcomes of candidemia in adults and children hospitalized in the United States: a propensity analysis. Clin. Infect. Dis. 41, 1232-1239 (2005).

31 Caliendo AM, Gilbert DN, Ginnochio CC et al. Better tests, better care: Improved diagnostics for infectious diseases. Clin. Infect. Dis. 57, S139-S170 (2013).

- Caliendo et al. summarize in this Infectious Diseases Society of America policy paper the current diagnostic landscape for infectious diseases, highlighting challenges to developing and implementing new tests, and presenting recommendations to address challenges at multiple levels.

32 Diekema DJ, Pfaller MA. Rapid detection of antibiotic-resistant organism carriage for infection prevention. Clin. Infect. Dis. 56, 1614-1620 (2013).

33 Holtzman C, Whitney D, Barlam T, Miller NS. Assessment of impact of peptide nucleic acid fluorescence in situ hybridization for rapid identification of coagulase-negative staphylococci in the absence of antimicrobial stewardship intervention. J. Clin. Microbiol. 49, 1581-1582 (2011).

34 Cleveland AA, Farley MM, Harrison LH et al. Changes in incidence and antifungal drug resistance in candidemia: results from population-based laboratory surveillance in Atlanta and Baltimore, 2008-2011. Clin. Infect. Dis. 55, 1352-1361 (2012).

35 Pfaller MA, Jones RN, Castanheira M. Regional data analysis of Candida nonalbicans strains collected in United States medical sites over a six-year period, 2006-2011. Mycoses 57, 602-611 (2014).

36 Pfaller MA, Andes DR, Diekema DJ et al. Epidemiology and outcomes of invasive candidiasis due to non-albicans species of Candida in 2, 496 patients: data from the Prospective Antifungal Therapy (PATH) registry 2004-2008. PLoS ONE 9, e101510 (2014). 